\title{
Recurrence rates after EMR of large sessile serrated polyps
}

Kevin D. Rex, Krishna C. Vemulapalli, Douglas K. Rex

\begin{abstract}
:
Background:

Little is known regarding the recurrence rate after endoscopic mucosal resection (EMR) of large

$(\geq 20 \mathrm{~mm})$ sessile serrated adenoma/polyps (SSA/Ps).
\end{abstract}

Objective:

Compare the rate of recurrence among SSA/Ps and conventional adenomas referred to a

specialty practice for EMR.

Design:

Retrospective cohort study

Setting:

Academic hospital and a satellite surgery center

Patients:

Three hundred and sixty two consecutive patients referred for resection of large ( $\geq 20 \mathrm{~mm})$ polyps

in the colorectum. 
Interventions:

All EMRs were performed with a submucosal contrast agent. All subjects had a follow-up surveillance exam (inspection and biopsy of the EMR) at our center.

Main outcome measurements:

Rates of residual polyp at follow-up examination.

Results:

Residual polyp was identified among $8.7 \%$ of SSA/Ps compared to $11.1 \%$ for conventional

adenomas $(\mathrm{p}=0.8)$.

Limitations:

Retrospective design; procedures performed by a single experienced endoscopist, low number of serrated lesions

Conclusions:

The rate of recurrence after EMR of SSA/Ps is similar to the rate following EMR of conventional adenomas. 
Introduction

Serrated histology has been identified as a predictor of incomplete polyp resection. In a prospective evaluation, predictors of incomplete resection included the endoscopist, increased polyp size, and sessile serrated adenoma/ polyp (SSA/P) histology (versus conventional adenoma $)^{1}$. The rate of incomplete resection among sessile serrated lesions overall was 31\%, versus $7 \%$ in conventional adenomas, and almost half of serrated lesions $10-20 \mathrm{~mm}$ in size were incompletely resected. However, the methods of polyp resection in that study were incompletely defined.

Serrated lesions (SSA/P and larger hyperplastic polyps) may be incompletely resected because the color of the lesions is similar to the surrounding mucosa, and the edges are indistinct ${ }^{2,3}$. Particularly during piecemeal resection, these factors may result in difficulty identifying the perimeter of the lesion and therefore incomplete resection. 
In this report, we describe the rate of residual polyp when SSA/Ps $\geq 20 \mathrm{~mm}$ in size were

removed by endoscopic mucosal resection using submucosal injection including a contrast agent

to define the perimeter of the lesion.

\section{Methods}

We prospectively maintain a database of sessile or flat lesions $\geq 20 \mathrm{~mm}$ in size resected by DKR.

Permission to review this database to determine rates of residual polyp and factors associated

with residual polyp was granted by the Institutional Review Board at Indiana University Health.

Inclusion criteria were lesion size at least $20 \mathrm{~mm}$, a pathology report of sessile serrated adenoma

or sessile serrated polyp or a conventional adenoma, resection between January 2006 and April

2014, first endoscopic follow-up performed at our institution, and that follow-up included biopsy

of the endoscopic mucosal resection (EMR) scar if the scar appeared endoscopically normal or

had only clip artifact. 
The primary end point was the rate of residual polyp detected at the first follow-up by either visual inspection of the polypectomy site and/or histologic sampling of the polypectomy scar.

Procedures were performed with high definition colonoscopes in the Olympus 180 series in most cases, and a few cases were performed with colonoscopes in the 160 or 190 series. The submucosal injection fluid early in the experience was saline or $10 \%$ dextrose and later hydroxyethyl starch. A contrast agent was included in all injections. Early in the experience the contrast agent was methylene blue and later indigo carmine. Snare resection was used for the entire polyp whenever possible. Snaring was initiated with stiff 20 to $33 \mathrm{~mm}$ diameter snares and sometimes completed using diminutive snares. Very flat portions of the lesions were sometimes snare resected with the assistance of a disposable transparent cap projecting a few mm past the end of the colonoscope (Olympus “distal attachment”; Olympus America Corp. Center Valley, PA). In these instances a flexible $11 \mathrm{~mm}$ snare (Captiflex, Boston Scientific, Natick MA) was placed over the flat tissue, and the tissue was suctioned partly or fully into cap, followed by snare closure until the tissue was felt in the snare (this was done "blindly" if the tissue was fully suctioned into the cap), followed by release of suction and then snare 
transection. Only after the potential for snare resection had been exhausted was ablation by argon plasma coagulation (APC) considered. APC was used focally to treat points on the perimeter and/or base of the EMR defect whenever there was any definite residual lesion (by analysis of pit patterns) that had defied snaring, or when there was any potential residual tissue (e.g. an uneven cut on the EMR edge or elevated points in the EMR base were often treated with APC even when no residual lesion pits were identifiable).

The first follow-up examination was performed 4-6 months after the EMR. Endoscopically identifiable polyp was removed by snare resection and sent for histologic evaluation, followed by APC of the perimeter of the snaring defect. Normal-appearing scars, or scars with only clip artifact, were subjected to systematic cold forceps biopsy of the entire length of the scar ${ }^{4}$. Statistical analyses

Descriptive information for the patients, polyps, and polypectomy techniques is presented with percentages for categorical variables and mean and standard deviations for continuous variables. Rates of residual polyp after EMR of sessile serrated polyps and conventional adenomas were 
compared using Fisher's exact test. A logistic regression analysis was performed to assess size, location, polyp pathology, resection type (piecemeal vs. en bloc), and use of APC on the presence of residual polyp at the first follow-up examination. We present odds ratios with $95 \%$ confidence intervals. All analyses were performed with the statistical software SPSS Version \#22 (IBM, New York, NY).

Results

There were 362 patients (196 males, 54.1\%) with 397 lesions (351 conventional adenomas and 46 SSA/Ps) that satisfied the inclusion criteria. Four of the SSA/Ps had cytological dysplasia reported. There were 10 additional lesions $\geq 20 \mathrm{~mm}$ in size interpreted by the pathologists as hyperplastic polyps which had followup at our center that included biopsies of the EMR site. One of these 10 lesions had endoscopically visible polyp on the EMR site at follow-up (confirmed by biopsy), 9 had no visible polyp and negative biopsies, and all 10 were excluded from the current analysis. 
Residual polyp at the first follow-up after EMR was identified in 43 cases (10.8\%), of which 32 cases (71\%) were evident endoscopically, and 12 (29\%) were identified only by cold biopsy of an endoscopically normal appearing EMR scar. Residual polyp was identified in 4 of the 46 SSA/Ps (8.7\%) vs. 39 of 351 conventional adenomas (11.1\%; $\mathrm{p}=0.8)$. SSA/Ps were more likely to be resected en bloc and less likely to require adjunctive treatment with APC (Table 1).

In univariate analyses, only size $(\mathrm{p}=0.02)$ predicted residual polyp at follow-up (Table 2$)$.

There were no significant differences in the age or sex of patients whose EMR sites had or did not have residual polyp. Logistic regression accounting for location, shape, and histology (Table 3) indicated that size alone predicted the presence of residual polyp at follow-up (OR 1.04; 95\%

CI 1.01, 1.06).

Discussion

In this study we demonstrate that rates of residual polyp at first follow-up after EMR of large SSA/Ps are similar to those after EMR of large conventional adenomas. All of the EMRs in this study were performed by a single endoscopist, but this is a potential advantage in understanding 
rates of residual polyp after serrated resection given recent evidence that the endoscopist is a

predictor of complete resection (1). The overall rates of residual polyp detected at first follow-up in this study are comparable to the lowest rates reported in EMR studies ${ }^{5,6}$. These data indicate that submucosal injection with a contrast agent combined with use of a high definition scope and an aggressive attempt at complete lesion snaring, followed by thermal destruction by APC of any definite residual lesion that escaped snaring (visible polyp glands) as well as frequent APC of potential residual lesion (mildly uneven points of the EMR defect edge or base were often treated by APC), is one technical approach that allows high rates of complete resection of large SSA/Ps. Two previous studies specifically examined cure rates at follow-up of SSA/Ps ${ }^{5,7}$. Only one of the two studies included a control group of conventional adenomas ${ }^{5}$, and only one utilized EMR for resection ${ }^{7}$. The current study is the only study to utilize both EMR and high definition examination and add forceps biopsy to maximize identification of residual polyp. Cold biopsy forceps have been shown in multiple studies to maximize detection of residual polyp ${ }^{4,8}$. We found that SSA/Ps, though of equal or numerically larger mean size than conventional adenomas, were more likely to be resected en bloc and less likely to receive any adjunctive APC compared to conventional adenomas. A previous study of EMRs of large sessile colorectal lesions also 
found that SSA/Ps were more often resected en bloc compared to conventional adenomas ${ }^{9}$.

Anecdotally, our impression is that SSA/Ps develop a more dome like shape with submucosal injection, and that stiff snares tend to slide less during closure onto SSA/Ps. In any case, in a multivariate analysis SSA/P histology did not predict a higher rate of recurrence after accounting for en bloc vs. piecemeal resection.

Our study has several limitations. First, the study was retrospective and subject to selection bias. However, the database we used to ascertain cases was prospectively assembled without reference to this study. Also we used well defined criteria (EMR with follow-up at our institution including biopsies of EMR scars) to identify all eligible cases. These methods should minimize selection bias. Second, the number of SSA/Ps in the study was small $(n=46)$ and there was an imbalance between the numbers of SSA/Ps and conventional adenomas, limiting the study power to assess factors such as lesion shape and location on recurrence rates, as well as technical factors such as piecemeal resection and use of ablation. Next, we used APC commonly at the end of the snaring phase of EMR, including cases where there were no endoscopically visible retained polyp glands but the edge or base of the EMR defect was uneven, and the contribution 
of APC to eradication of either the SSA/Ps or the conventional adenomas is uncertain. Despite that, APC was used more often in the conventional adenomas than the SSA/Ps, suggesting that submucosal injection with a contrast agent and snare resection is as effective in SSA/Ps as in conventional adenomas. Next, we used the routine pathology reports to include SSA/Ps and exclude large hyperplastic polyps, though there is substantial interobserver variation in this designation by pathologists ${ }^{10-12}$. However, the recurrence rate in EMR sites of the 10 large hyperplastic polyps we excluded was $10 \%$, and inclusion of some or all of these lesions in the SSA/P group would not have changed the study conclusion. Finally, the results shown here reflect colonoscopy by a single experienced operator and may not be generalizable.

In summary, we found that EMR of large SSA/Ps performed with a submucosal contrast agent and high definition colonoscope leads to high endoscopic cure rates at first follow-up that are comparable to those achieved with conventional adenomas. Endoscopists should not hesitate to undertake EMR of large SSA/Ps because of concerns identified in previous studies that SSA/Ps are associated with a high risk of incomplete resection. 


\section{References:}

1. Pohl H, Srivastava A, Bensen SP, et al. Incomplete polyp resection during colonoscopy-results of the complete adenoma resection (CARE) study. Gastroenterology 2013;144:74-80 e1.

2. Tadepalli US, Feihel D, Miller KM, et al. A morphologic analysis of sessile serrated polyps observed during routine colonoscopy (with video). Gastrointest Endosc 2011;74:1360-8.

3. Hazewinkel Y, Lopez-Ceron M, East JE, et al. Endoscopic features of sessile serrated adenomas: validation by international experts using high-resolution white-light endoscopy and narrowband imaging. Gastrointest Endosc 2013;77:916-24.

4. Khashab M, Eid E, Rusche M, et al. Incidence and predictors of "late" recurrences after endoscopic piecemeal resection of large sessile adenomas. Gastrointest Endosc 2009;70:344-9.

5. L Liang J, Kalady MF, Church J. Snaring large serrated polyps. Surg Endosc 2013;27:1622-7.

6. Moss A, Williams SJ, Hourigan LF, et al. Long-term adenoma recurrence following wide-field endoscopic mucosal resection (WF-EMR) for advanced colonic mucosal neoplasia is infrequent: results and risk factors in 1000 cases from the Australian Colonic EMR (ACE) study. Gut 2014.

7. Pellise M, Burgess, N.G., Tuttici, N.J., Hourigan, L.F., Zanati, S.A, Brown, G.J., Singh, R., Williams, S.J., Raftopoulos, S.C., Moss, A., Ormonde, D., P'NG, C.H, Mcleod, D.J., Bourke, M.J. 613 Large Sessile Serrated Adenomas: Outcome of Wide Field Endoscopic Mucosal Resection (Wf-EMR) in a Multicenter Prospective Cohort. Gastrointest Endosc 2014;79:AB160-AB161.

8. Knabe M, Pohl J, Gerges C, et al. Standardized long-term follow-up after endoscopic resection of large, nonpedunculated colorectal lesions: a prospective two-center study. Am J Gastroenterol 2014;109:183-9.

9. Burgess NG, Nanda KS, Williams SJ, et al. 505 Comparison of Large Sessile Serrated Adenoma Characteristics With Conventional Advanced Mucosal Neoplasia Resected by Wide Field Endoscopic Mucosal Resection in a Multicenter Prospective Cohort. Gastrointestinal Endoscopy;77:AB157.

10. Khalid O, Radaideh S, Cummings OW, et al. Reinterpretation of histology of proximal colon polyps called hyperplastic in 2001. World J Gastroenterol 2009;15:3767-70.

11. Payne SR, Church TR, Wandell M, et al. Endoscopic detection of proximal serrated lesions and pathologic identification of sessile serrated adenomas/polyps vary on the basis of center. Clin Gastroenterol Hepatol 2014;12:1119-26.

12. Abdeljawad K, Vemulapalli KC, Kahi CJ, et al. Sessile serrated polyp prevalence determined by a colonoscopist with a high lesion detection rate and an experienced pathologist. Gastrointest Endosc 2014. 
Table 1 Differences between conventional adenomas and sessile serrated adenoma/polyps

\begin{tabular}{|l|l|l|l|}
\hline & $\begin{array}{l}\text { SSA/P histology, } \\
\mathrm{n}=46\end{array}$ & $\begin{array}{l}\text { Conventional } \\
\text { adenoma histology, } \\
\mathrm{n}=351\end{array}$ & $\mathrm{p}$-value \\
\hline Residual rate & $4(8.7)$ & $39(11.1)$ & $0.8^{*}$ \\
\hline Size in mm, mean (SD) & $32.6(10.3)$ & $31.1(11)$ & 0.36 \\
\hline Sessile Shape (vs flat) & $36(78.3)$ & $244(69.5)$ & 0.22 \\
\hline Right colon location (vs left)† & $37(80.4)$ & $216(61.5)$ & 0.01 \\
\hline $\begin{array}{l}\text { Piecemeal polypectomy (vs en } \\
\text { bloc) }\end{array}$ & $35(76.1)$ & $307(87.5)$ & 0.04 \\
\hline Ablation used (vs no ablation) & $34(73.9)$ & $305(86.9)$ & 0.02 \\
\hline
\end{tabular}

*Fisher's exact test

tRight is proximal to descending colon

SD: standard deviation

SSA/P: sessile serrated adenoma/polyp 
Table 2 Characteristics of EMR sites with residual polyp and EMR scars with no endoscopically or histologically detectable polyp

\begin{tabular}{|l|l|l|l|}
\hline Index polyp characteristic & $\begin{array}{l}\text { Residual polyp (\%) } \\
n=43\end{array}$ & $\begin{array}{l}\text { No detectable polyp (\%) } \\
n=354\end{array}$ & $\mathrm{p}$-value \\
\hline Histology (adenoma/SSA-P) & $39 / 4(90.7 / 9.3)$ & $312 / 42(88.1 / 11.9)$ & $0.8^{*}$ \\
\hline Size in mm, mean (SD) & $34.8(11.1)$ & $30.8(10.8)$ & 0.02 \\
\hline Shape (sessile/flat) & $28 / 15(65.1 / 34.9)$ & $252 / 102(71.2 / 28.8)$ & 0.41 \\
\hline Location (right/left) & $29 / 14(67.4 / 32.6)$ & $224 / 130(63.3 / 36.7)$ & 0.59 \\
\hline $\begin{array}{l}\text { Piecemeal polypectomy vs. } \\
\text { en bloc (yes/no) }\end{array}$ & $38 / 5(88.4 / 11.6)$ & $304 / 50(85.9 / 14.1)$ & 0.66 \\
\hline Ablation used (yes/no) & $36 / 7(83.7 / 16.3)$ & $303 / 51(85.6 / 14.4)$ & 0.74 \\
\hline
\end{tabular}

*Fisher's exact test

tRight is proximal to descending colon

EMR: endoscopic mucosal resection

SD: standard deviation

SSA/P: sessile serrated adenoma/polyp 
Table 3 Regression analysis for the presence of residual polyp at first follow-up

\begin{tabular}{|l|l|l|}
\hline Risk factor at index lesion & OR $(95 \% \mathrm{Cl})$ & $\mathrm{p}$-value \\
\hline Size (increments of $1 \mathrm{~mm})$ & $1.04(1.01-1.06)$ & 0.01 \\
\hline Shape (sessile/flat) & $0.66(0.33-1.32)$ & 0.24 \\
\hline Histology (SSA/P/adenoma) & $0.67(0.22-2.05)$ & 0.49 \\
\hline Location (right/left) & $1.45(0.72-2.94)$ & 0.3 \\
\hline $\begin{array}{l}\text { Piecemeal polypectomy vs en } \\
\text { bloc resection (yes/no) }\end{array}$ & $1.13(0.4-3.3)$ & 0.82 \\
\hline Ablation used (yes/no) & $0.71(0.28-1.79)$ & 0.47 \\
\hline
\end{tabular}

tRight is proximal to descending colon

OR: odds ratio

SSA/P: sessile serrated adenoma/polyp 